\title{
Conditions de vie dans les territoires contaminés en Biélorussie 8 ans après l'accident de Tchernobyl*
}

\author{
Évaluation de la situation dans le district \\ de Tchetchersk en Biélorussie
}

G. HÉRIARD-DUBREUIL**, P. GIRARD***

(Manuscrit reçu le 19 février 1996, révisé le 27 septembre 1996, accepté le 20 février 1997)

RÉSUMÉ Cet article présente une analyse des enjeux sociaux et économiques de la protection radiologique dans les territoires contaminés du fait de l'accident de Tchernobyl. Il s'appuie sur les résultats de deux enquêtes réalisées en 1994 sur les conditions de vie des habitants d'une collectivité territoriale située en Biélorussie à $180 \mathrm{~km}$ au nord de Tchemobyl. Une première partie présente les données radiologiques de la situation post-accidentelle du district avec ume analyse de son impact démographique depuis 1986. Une seconde partie présente les enjeux de la protection radiologique avec une description des modalités d'exposition des habitants des territoires contaminés et une évaluation des différents programmes de contre-mesures engagés par les autorités, dans le cadre législatif de 1991. Dans une dernière partie sont présentés les aspects économiques de la gestion post-accidentelle avec une analyse du budget du district de Tchetchersk et une évaluation des conséquences de la situation radiologique sur les activités économique et surtout agricole de ce district. La conclusion fait ressortir les difficultés auxquelles sont aujourd'hui confrontées les autorités biélorusses. La présence désormais définitive de populations dans un environnement durablement contaminé pose une nouvelle catégorie de problèmes. Les objectifs de radioprotection doivent être resitués dans un ensemble de contraintes de différentes natures, notamment sociales et économiques. Le développement de la sécurité radiologique est indissociable du retour d'une qualité de vie dans ces territoires. I passe nécessairement par le redéploiement d'un climat de confiance sociale. Le dispositif législatif initial de gestion post-accidentelle doit être adapté pour donner plus d'autonomie aux acteurs locaux dans la reconstruction de conditions de vie satisfaisantes.

ABSTRACT Living conditions in the contamingted territories of Byelorussia after the Chernobyl accident : evaluation of the situation in the district of Chetchersk in Byelorussia.

This article presents an analysis of the social and economic aspects of radiological protection in the territories contaminated by the Chemobyl accident. It is based on the results of two surveys performed in 1994 on the living conditions

\footnotetext{
* Les travaux qui sont à l'origine de cet article ont été réalisés avec le soutien de la Commission Européenne, dans le cadre de ses actions de recherche en radioprotection.

** Mutadis consultants, 14 rue de Belzunce, 75010 Paris.

*** Université de Caen.
} 
of the inhabitants of a territorial community located in Byelorussia, $180 \mathrm{~km}$ north of Chernobyl. The first part presents the radiological post-accident situation of the district, together with an analysis of this situation's demographic impact since 1986. The second part presents a description of the modes of exposure of the inhabitants of the contaminated territories and an assessment of the various countermeasures programmes initiated by the authorities in the legislative framework of 1991. The last part addresses the economic aspects of the post-accident management, together with an analysis of the budget of the Chetchersk district and an evaluation of the consequences of the radiological situation on the economic, and above all agricultural, activities of the district. The conclusion highlights the difficulties that face the Byelorussian authorities today. The now definitive presence of inhabitants in a durably contaminated environment poses a new category of problems. The objectives of radiological protection have to be reshaped within a set of constraints of different types, notably social and economic. The development of radiological safety cannot be dissociated from a return to quality living in these territories. This necessarily entails re-establishing a climate of social confidence. The initial legislative plan for post-accident management must be adapted to give greater autonomy to local participants in the reconstruction of satisfactory living conditions.

\section{Introduction}

De toutes les Républiques de l'ancienne Union Soviétique, la Biélorussie est la plus touchée par l'accident de Tchernobyl avec $23 \%$ de son territoire contaminé, soit une surface d'environ $46500 \mathrm{~km}^{2}$. A titre de comparaison, l'Ukraine (Girard \& Hériard Dubreuil, 1994 ; Hériard Dubreuil, 1994) n'a que 4,8\% de son territoire contaminé et la Russie 0,5\%. En Biélorussie, les régions les plus contaminées sont celles de Gomel, Mogilev et Brest, essentiellement situées au sud et sud-est de la République. Plus de 2 millions de Biélorusses vivent dans des territoires contaminés radiologiquement à des degrés divers, soit un cinquième de la population biélorusse totale qui est de 10,3 millions d'habitants.

A partir de 1991, le Parlement et les autorités biélorusses ont mis en place un dispositif législatif qui détermine les conditions de la gestion post-accidentelle ainsi que les modalités de réparation des dommages accidentels pour les différentes catégories de personnes concernées par cet accident. La disparition de l'Union Soviétique, depuis 1991, a provoqué une crise sans précédent dans l'économie biélorusse auparavant prospère mais totalement dépendante de l'ancien système économique soviétique. Cette crise économique est venue s'ajouter aux difficultés rencontrées par les populations affectées par l'accident.

Cet article s'appuie sur les résultats de deux enquêtes réalisées en 1994 sur les conditions de vie des habitants des territoires contaminés dans le cadre du projet JSP-2 du « Programme international d'évaluation des conséquences de l'accident nucléaire de Tchernobyl » développé par la Commission européenne, en collaboration avec la Communauté des États indépendants (CEI). Il présente une analyse des enjeux sociaux et économiques de la protection 
radiologique à l'échelle d'une collectivité territoriale biélorusse, le district de Tchetchersk, situé à $180 \mathrm{~km}$ au nord de Tchernobyl, dans les territoires contaminés de la région de Gomel.

Une première partie présente les données radiologiques de la situation post-accidentelle du district avec une analyse de son impact démographique depuis 1986. Une seconde partie présente les enjeux de la protection radiologique avec une description des modalités d'exposition des habitants des territoires contaminés et une évaluation des différents programmes de contremesures engagés par les autorités, dans le cadre législatif de 1991. Dans une dernière partie sont présentés les aspects économiques de la gestion post-accidentelle avec une analyse du budget du district de Tchetchersk et une évaluation des conséquences de la situation radiologique sur les activités économique et surtout agricole de ce district. La conclusion fait ressortir les difficultés auxquelles sont aujourd'hui confrontées les autorités biélorusses dans la gestion post-accidentelle et notamment le caractère inadapté du cadre législatif élaboré en 1991. Les objectifs de radioprotection doivent nécessairement être resitués dans un ensemble de contraintes sociales et économiques. La sécurité radiologique est indissociable d'une amélioration globale des conditions de vie dans les territoires contaminés.

\section{Méthodologie des enquêtes sur le terrain}

Les évaluations de la situation post-accidentelle présentées dans cet article ont été réalisées à partir d'éléments recueillis auprès de l'administration nationale biélorusse et sur le terrain dans les territoires contaminés, dans le cadre de deux missions en mars et en juillet 1994. Les données ont été recueillies dans le cadre d'entretiens avec les autorités nationales et locales ainsi qu'avec les principaux experts biélorusses et auprès de la population des territoires contaminés. Ces données ont été complétées par des informations tirées de différents documents rassemblés dans le cadre de ces missions ou provenant d'études fournies par le Comité d'État Tchernobyl de Biélorussie. D'autres sources ont également été utilisées (études UNIDO). Les informations recueillies présentaient parfois certaines lacunes ou incohérences. Un travail de recoupement entre les différentes sources locales, nationales et internationales a néanmoins permis de dresser un premier tableau de la situation analysée.

Durant la mission d'étude de mars 1994, une quarantaine d'interviews de responsables et d'experts impliqués à différents niveaux (national, régional et local) ont apporté une première évaluation des enjeux de la gestion post-accidentelle en Biélorussie, près de huit ans après l'accident de Tchernobyl. Dans une seconde mission réalisée en juillet 1994, une enquête a été réalisée dans le district de Tchetchersk, situé dans l'une des zones les plus contaminées de Biélorussie, pour évaluer de façon précise, auprès des autorités et auprès de la population, les conditions de vie dans un environnement durablement contaminé et les enjeux de la gestion du risque radiologique dans ce contexte. Cette 
évaluation a été faite dans le cadre d'une approche qualitative (entretiens semidirectifs) auprès d'une centaine de personnes sélectionnées au hasard sur les différents lieux d'investigation (centre du district, villages, centres de production).

Les entretiens ont été réalisés auprès de catégories de population sélectionnées en fonction de leur implantation géographique dans le district (centre administratif, village). L'échantillonnage des villages a été fait en tenant compte du zonage administratif du niveau de contamination. Des entretiens ont parallèlement été conduits auprès des différentes autorités impliquées dans la gestion du risque radiologique (autorités du district, médecins, experts en radioprotection, administration vétérinaire, responsables de centres de production, etc). Des entretiens avec des adhérents de différentes associations liées à l'accident ont également été réalisés. Les investigations sur le terrain ont été menées de façon systématique, sur la base d'une sélection aléatoire, sans aucune préparation préalable, auprès des populations relogées ainsi que dans les différentes zones de contamination, soit (pour le ${ }^{137} \mathrm{Cs}$ ) de 1 à $5 \mathrm{Ci} / \mathrm{km}^{2}$ $\left(37-185 \times 10^{9} \mathrm{~Bq} / \mathrm{km}^{2}\right)$, de 5 à $15 \mathrm{Ci} / \mathrm{km}^{2}\left(185-555 \times 10^{9} \mathrm{~Bq} / \mathrm{km}^{2}\right)$, plus de $15 \mathrm{Ci} / \mathrm{km}^{2}$ (plus de $555 \times 10^{9} \mathrm{~Bq} / \mathrm{km}^{2}$ ).

\section{Situation générale du district de Tchetchersk en 1994(1)}

\subsection{Un district fortement contaminé}

Le district de Tchetchersk est situé au sud-est de la Biélorussie dans la région (oblast) de Gomel (à $60 \mathrm{~km}$ au nord de Gomel, près de la frontière de la Russie). Sa surface est de 120000 hectares. Sur un plan administratif, il est divisé en 7 soviets. La ville de Tchetchersk est le centre administratif du district. Elle est située à $180 \mathrm{~km}$ environ de Tchernobyl.

Le territoire du district de Tchetchersk est fortement contaminé du fait de l'accident de Tchernobyl. Avant cet accident, le district comptait 47900 hectares de terres agricoles. Une proportion de près de $24 \%$ de cette surface, c'est-à-dire 11500 hectares environ, est contaminée à plus de $40 \mathrm{Ci} / \mathrm{km}^{2}$. La culture y est aujourd'hui interdite. La majorité des terres cultivables (90\%) est contaminée à plus de $5 \mathrm{Ci} / \mathrm{km}^{2}$ (Fig. 1).

Près de $50 \%$ du territoire du district se composent de forêts dont seulement $15 \%$ se situent dans la zone de contamination inférieure à $5 \mathrm{Ci} / \mathrm{km}^{2}$ (zone la moins contaminée) et $6 \%$ dans un territoire contaminé à plus de $40 \mathrm{Ci} / \mathrm{km}^{2}$ (zone la plus contaminée).

(1) Ce chapitre a été élaboré en tenant compte de données fournies par les autorités de district de Tchetchersk, ou recueillies dans le cadre d'entretiens avec des experts et des membres de l'administration, ou provenant de d'études fournies par le Comité d'état Tchernobyl de Biélorussie. 
La population du district, selon les autorités, est aujourd'hui proche de 18500 habitants dont près d'un tiers habite la ville de Tchetchersk. Il y a aujourd'hui près de 110 villages habités dans le district. La répartition de la population selon le niveau de contamination montre que la population réside essentiellement dans des territoires contaminés à plus de $5 \mathrm{Ci} / \mathrm{km}^{2}$ (Fig. 2).

\begin{tabular}{|c|c|c|c|}
\hline Categorie de zone & $\begin{array}{l}\text { Cogtamination en } \\
\text { Cuntr }^{2} \text { (Cesium) }\end{array}$ & $\begin{array}{l}\text { Contamination } \\
\text { des territoires agricoles } \\
\text { di distriet de Tchetchers }\end{array}$ & $\begin{array}{l}\text { Dose annuele } \\
\text { en mSv }\end{array}$ \\
\hline \multicolumn{4}{|c|}{ Zone 1 « d'évacuation » } \\
\hline $\begin{array}{l}\text { Zone } 2 \text { "relogement } \\
\text { prioritaire " }\end{array}$ & $40^{(1)}$ & $24 \%$ & \\
\hline $\begin{array}{l}\text { Zone } 3 \text { « relogement } \\
\text { ultérieur }\end{array}$ & $15-40$ & $17 \%$ & $>5$ \\
\hline $\begin{array}{l}\text { Zone } 4 \text { 《droit } \\
\text { au relogement » }\end{array}$ & $5-15$ & $49 \%$ & 1 à 5 \\
\hline $\begin{array}{l}\text { Zone } 5 \text { « contrôle } \\
\text { radiologique » }\end{array}$ & $1-5$ & $10 \%$ & $\leq 1$ \\
\hline
\end{tabular}

Fig. I. - Contamination des terres agricoles du district de Tchetcherk, répartition en pourcentages selon les catégories légales de zones contaminées, d'après le dispositif légal biélorusse.

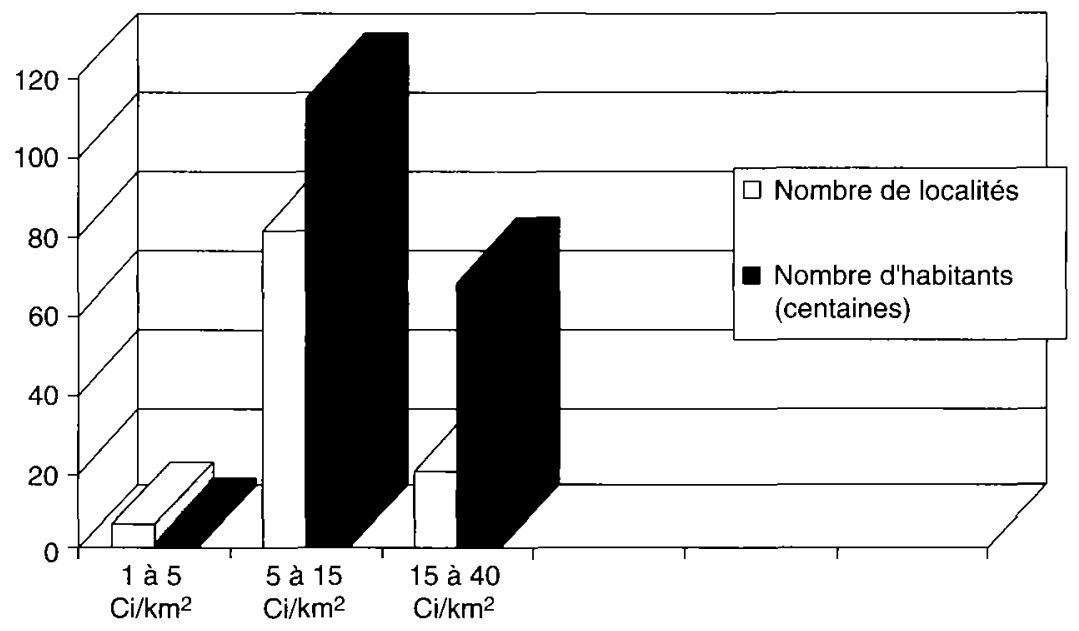

Fig. 2. - Répartition de la population selon le niveau de contamination. District de Tchetchersk. 
La plus grande partie de la population se trouve en effet dans des zones initialement promises au relogement, selon les termes de la loi biélorusse. Les territoires dont la contamination est supérieure à $40 \mathrm{Ci} / \mathrm{km}^{2}$ (zone dite "d'évacuation » dans la loi biélorusse) sont aujourd'hui pratiquement évacués. Dans la zone dont la contamination se situe entre 15 et $40 \mathrm{Ci} / \mathrm{km}^{2}$ (dite « de relogement prioritaire "), se trouvent 19 villages ainsi que la ville de Tchetchersk, soit un total de 6769 habitants (sans compter près de 5000 résidents temporaires de Tchetchersk qui ne sont pas pris en compte dans les données officielles voir plus loin).

Dans la zone dont la contamination se situe entre 5 et $15 \mathrm{Ci} / \mathrm{km}^{2}$ (dite « avec le droit au relogement »), se trouvent 81 villages et 11419 habitants. Dans la zone dont la contamination se situe entre 1 et $5 \mathrm{Ci} / \mathrm{km}^{2}$ (dite « de résidence avec contrôle radiologique périodique »), se trouvent 6 villages et 157 habitants. Dans la zone 4 (de 5 à $15 \mathrm{Ci} / \mathrm{km}^{2}$ ), le nombre moyen d'habitants par village est de 140 personnes. Dans la zone 3 (de 15 à $40 \mathrm{Ci} / \mathrm{km}^{2}$ ), le nombre moyen d'habitants par village (en excluant la ville de Tchetchersk) est de 49 personnes. On voit donc que, dans la zone habitée la plus contaminée, la population est plus dispersée que dans les autres zones. Cette répartition de la population pose problème dans la mesure où elle contribue à diluer les moyens disponibles pour créer un environnement et des infrastructures adaptés à ce niveau de contamination. Les forêts, qui couvrent plus de la moitié du territoire du district, sont fortement et durablement contaminées. Les experts biélorusses estiment que les localités proches des forêts constituent un environnement très défavorable sur le plan de la radioprotection et difficilement maîtrisable.

\subsection{La population du district a diminué de près d'un tiers depuis 1986}

La ville de Tchetchersk se trouve dans une zone dont le niveau de contamination moyen est évalué à $16 \mathrm{Ci} / \mathrm{km}^{2}$. De ce fait, étant contaminée à plus de $15 \mathrm{Ci} / \mathrm{km}^{2}$, cette ville est située dans la zone administrative $n^{\circ} 3$, dite de « relogement ultérieur » et devait initialement être entièrement évacuée. L'évacuation de la ville de Tchetchersk est aujourd'hui différée sine die suite à un décret du Parlement biélorusse, mais cette décision n'est pas encore définitivement confirmée.

La population du district a diminué de près d'un tiers depuis l'accident de Tchernobyl, du fait du départ d'une partie des habitants vers des zones non contaminées. Différentes mesures de relogement ont été mises en place depuis 1986, mais surtout depuis 1991 avec la promulgation des lois post-accidentelles. L'évolution de la population du district est présentée en figure 3.

Les autorités considèrent que la population du district est aujourd'hui en voie de stabilisation, ce que confirment les interviews sur le terrain. De même, on note, depuis 1990, un mouvement d'immigration dans le district en provenance d'autres républiques de l'ex-URSS. Ces personnes sont généralement poussées à quitter leur lieu initial de résidence pour des raisons diverses 


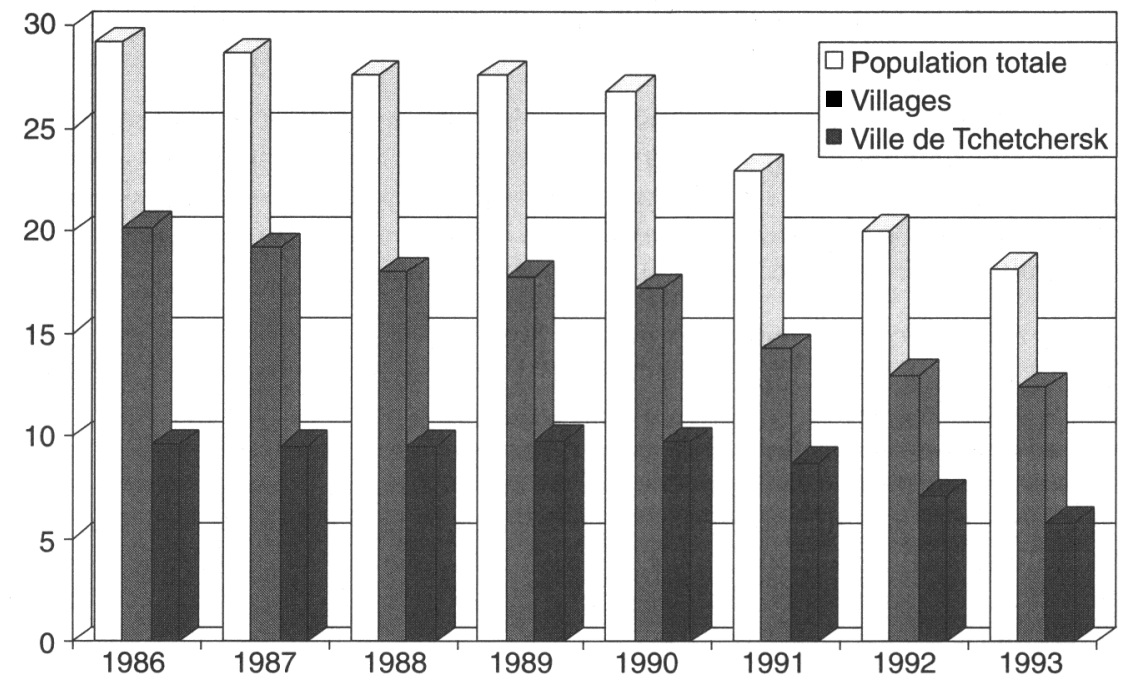

Fig. 3. - Population du district de Tchetchersk, en milliers, au total, en zone rurale (villages) et dans la ville de Tchetchersk.

(guerre, problèmes ethniques et nationaux, crise économique) qui les conduisent à venir trouver refuge dans ce district. Ainsi, en 1993, 554 personnes (principalement originaires du Kazakhstan, de Russie, d'Ukraine, d'Ouzbekhistan) sont venues s'installer dans le district. Leur nombre reste faible, au regard de l'ensemble de la population.

La figure 3 ne prend toutefois en compte que la population résidant officiellement dans le district. Dans les villages, le nombre des habitants correspond effectivement au nombre des inscrits. Dans la ville de Tchetchersk, en revanche, on peut estimer, d'après les autorités, qu'en plus de la population résidant officiellement (5800 personnes en 93) il faut compter près de 5000 habitants qui ne sont pas officiellement inscrits comme résidents permanents en ville, mais y résident temporairement à titre professionnel (et résident officiellement dans un autre district). Les autorités estiment que le nombre réel d'habitants du centre du district est donc à peu près identique en 1993 à celui de 1986 (de l'ordre de 10000 habitants).

\subsection{La population des "non résidents" de la ville de Tchetchersk}

Cette population de non-résidents (5 000 personnes, près de la moitié de la population initiale de la ville) est composée essentiellement d'anciens habitants de Tchetchersk qui ont bénéficié des dispositions légales post-accidentelles. Ils ont tout d'abord déménagé hors du district et reçu un logement dans une autre 
ville de la République, en dehors des territoires contaminés ou dans des territoires moins contaminés.

Pour faire face aux difficultés soulevées par le départ de la population active et particulièrement des personnes disposant d'une qualification (médicale, technique, administrative), la loi prévoit un système contractuel permettant à certaines catégories de travailleurs exerçant leur profession dans une zone contaminée de bénéficier d'un surcroît de salaire.

Ces dispositions ont conduit certains habitants de Tchetchersk à demander d'abord leur relogement pour bénéficier d'un nouvel appartement (pour euxmêmes ou leurs enfants) puis à réintégrer leur logement de Tchetchersk pour y exercer leur emploi initial laissé vacant, parfois avec un double salaire, avantage important du fait de la sévérité de la crise économique et de la crainte du chômage. On peut donc observer que si le nombre d'habitants de la ville de Tchetchersk est équivalent en 1994 à celui de 1986, c'est en grande partie à cause de ces dispositions post-accidentelles(1) (pseudo relogement et système contractuel).

Ce système contractuel n'est accessible qu'aux non-résidents. Ainsi les habitants de Tchetchersk qui disposent d'une qualification, mais qui n'ont pas pu bénéficier du relogement, sont donc doublement pénalisés dans la mesure où ils ne peuvent bénéficier du système de contrat étant encore résidents de Tchetchersk. Cette mesure provoque des frustrations dans les couches de la population qui n'y ont pas accès. De plus, l'existence de gratifications justifiée par le niveau de contamination constitue une sorte de confirmation implicite du risque associé à la résidence dans le district.

\subsection{Le vieillissement de la population des campagnes}

Dans les zones rurales, ce sont d'abord les familles avec des enfants qui ont été relogées en priorité. Les départs dans les villages n'ont pas été compensés par des retours de population sous contrat, comme dans la ville de Tchetchersk. De ce fait, on constate un fort vieillissement de la population rurale de ce district. Le nombre des enfants a fortement diminué dans les villages depuis 1986 (le nombre d'habitants a diminué de $40 \%$ et le nombre des enfants de $50 \%$ ), tandis qu'en ville, leur nombre semble s'être à peu près maintenu (dans le centre du district la population des résidents chute de $40 \%$ tandis que le nombre des enfants n’a chuté que de $20 \%$, Fig. 4).

(1) Dans le cas des populations disposant d'une qualification technique, qui ne sont pas originaires du district, le système de contrat est clairement un facteur dominant de motivation à vivre dans le district de Tchetchersk. Dans le cas des populations originaires du district, qui sont revenues s'y installer aprés l'avoir quitté, le système de contrat peut constituer un facteur de motivation parmi d'autres (volonté de conserver le logement initial, proximité de la famille, attrait du sol natal). Le système de contrat n'est cependant accessible qu'à certaines catégories de techniciens. 


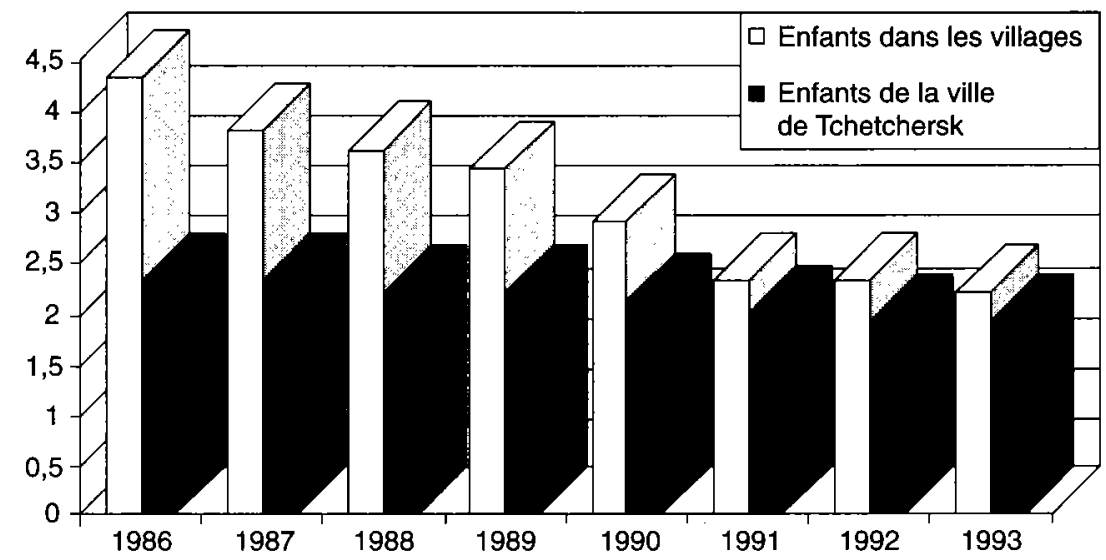

Fig. 4. - Population du district de Tchetchersk, en milliers, nombre d'enfants (0-17 ans) en zone rurale (villages) et dans la ville de Tchetchersk.

\subsection{L'évolution de la natalité et de la mortalité dans le district}

Les statistiques du district de Tchetchersk indiquent une augmentation régulière de la mortalité depuis 1986, ceci particulièrement en milieu rural, dans les villages. Il est difficile d'interpréter ces chiffres en l'absence de données médicales précises. Il faut toutefois noter que ce phénomène est probablement lié, au moins pour une part, au vieillissement de la population, surtout en milieu rural.

On observe depuis 1986, une constante diminution de la natalité dans les villages jusqu'en 1992, année à partir de laquelle on constate une légère remontée du taux de natalité en ville comme dans les villages. D'une façon générale, on observe que l'évolution des taux de mortalité et de natalité est assez différente en milieu rural et en milieu urbain (Figs. $5 \& 6$ ).

En 1993, le taux de mortalité dans les villages (25,3 pour mille) est près du double du taux de natalité ( 12,9 pour mille). Le taux de mortalité en ville (16,1 pour mille) est largement inférieur au taux de natalité en ville $(25,1$ pour mille). On constate donc des différences importantes entre les données urbaines et rurales. Ainsi en 1993, le taux de natalité dans la ville de Tchetchersk est près du double de celui des villages (notons que les taux de mortalité et de natalité en 1986 sont déjà très différents en milieux rural et urbain). De même, le taux de mortalité dans les villages est beaucoup plus important qu'en ville. 


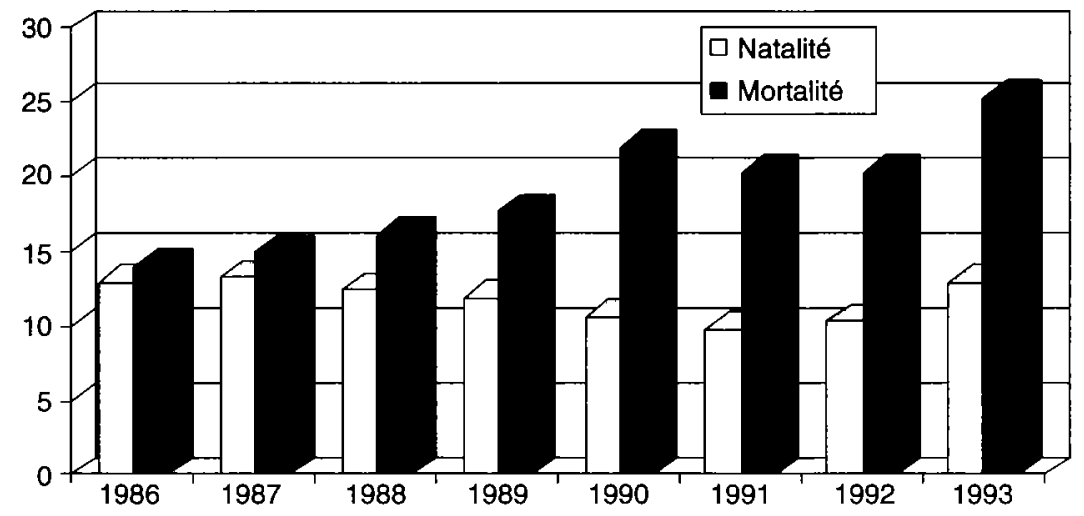

Fig. 5. - Taux de natalité et de mortalité en zone rurale (villages) dans le district de Tchetchersk (pour 1 000).

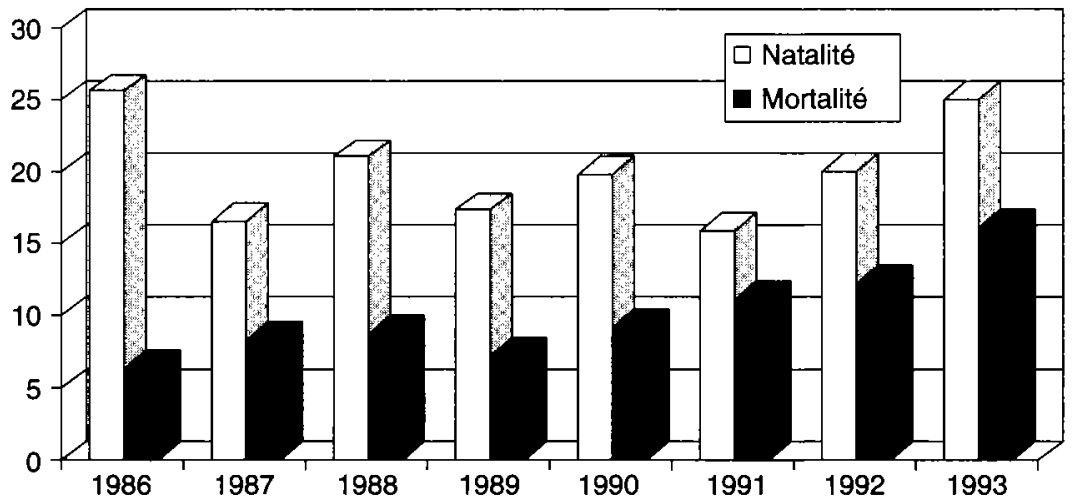

Fig. 6. - Taux de natalité et de mortalité dans la ville de Tchetchersk (pour 1 000).

D'une façon générale ces différences illustrent les impressions recueillies sur le terrain : une population vieillissante avec peu d'enfants dans les campagnes et une vie sociale en régression. Au contraire, en ville, une population plus jeune avec une majorité d'actifs et la présence de nombreux enfants. On remarquera que le taux de mortalité en ville a augmenté en proportion plus sensiblement que dans les villages, ce qui semble a priori paradoxal. On peut supposer que cette différence est articielle et résulterait en réalité d'une absence de prise en compte de la population des non résidents dans le calcul du taux de mortalité (les décès seraient enregistrés, y compris pour la population croissante des non résidents, tandis que le ratio de mortalité serait calculé sur la base constante des inscrits). 


\section{La gestion de la situation radiologique dans le district de Tchetchersk}

\subsection{Les modes d'exposition de la population}

Compte tenu du niveau de contamination des territoires du district de Tchetchersk, la grande majorité du district devait être initialement relogée. En 1994, force est de constater la présence de près de deux tiers de la population initiale dans les villages du district d'une part et la présence d'un nombre d'habitants équivalent à 1986 dans la ville de Tchetchersk d'autre part. Pour différents motifs (chômage, pénurie alimentaire, attachement à la terre natale, etc.), le mouvement d'émigration semble être aujourd'hui en voie d'achèvement. L'objectif des autorités est donc aujourd'hui d'assurer à la population des conditions de vie satisfaisantes au plan sanitaire et économique, qui sont d'ailleurs indissociables. Les conditions de vie sont en effet problématiques. Cependant, la volonté de privilégier la productivité économique du district peut conduire les autorités locales à négliger la protection radiologique (ce qui semble être la tendance actuelle).

La crise économique et l'inflation depuis 1991 ont provoqué une régression de la protection radiologique. La production alimentaire privée qui avait été initialement réduite à cause de son niveau de contamination, a largement repris. Dans les années qui ont suivi l'accident, les habitants du district avaient presque cessé la production laitière (considérée comme trop contaminée) et cédé leurs vaches sous la pression des autorités. Les difficultés économiques ont modifié radicalement cette situation. Ainsi, la plupart des familles ont racheté des vaches pour reprendre une production laitière largement contaminée.

L'exposition et la contamination radiologique des habitants du district s'effectuent à travers de multiples vecteurs que sont tous les actes de la vie quotidienne. La contamination provient d'abord de l'alimentation, que celle-ci soit d'origine privée (lait, viande, produits forestiers, gibier ou poisson, miel, légumes de potager) ou d'origine publique(1). L'exposition et la contamination radiologique s'effectuent encore à travers l'exercice des activités professionnelles (agricoles pour la plupart des actifs) ou à travers des activités personnelles ou de loisir : activités domestiques intérieures ou de plein air, transport, chauffage, sport (promenades, baignades), etc. La contamination provient également de la circulation de poussières contaminées. Dans le district, on compte

(1) Nous parlerons de circuit public par opposition au circuit privé (potagers, fermes, élevage domestique), par convention, dans la mesure où il est encore difficile de parler de secteur économique indépendant des autorités dans le contexte actuel de la Biélorussie. Les magasins où sont distribués les produits relèvent de l'autorité administrative, comme certains centres de production (sofkhoses). Les kolkhoses, en revanche, ont un caractère coopératif et pourraient assez rapidement gagner une certaine indépendance. De même le dispositif de contrôle radiologique est indirectement contrôlé par les autorités du district et n'est donc pas indépendant. 
que près de $70 \%$ des habitants se chauffent au bois provenant de la forêt qui est largement contaminée. De même, près de $30 \%$ des localités n'ont aucune route asphaltée.

L'alimentation des habitants est essentiellement produite dans le district, par le circuit privé (fermes, potagers) et par les entreprises (sovkhozes et kolkhozes) dont une partie de la production est distribuée dans les 6 magasins de la ville de Tchetchersk ainsi que par les magasins de village (le reste est exporté). La dégradation du contexte économique et l'inflation font que la population des villages n'a pratiquement pas accès au circuit public et s'alimente donc essentiellement à partir de sa propre production, complétée, dans les localités proches de la forêt, par des produits forestiers ou des produits de la pêche.

Même en ville, la plupart des habitants ont un potager et élèvent des animaux (vaches, porcs, volailles) pour compléter leur alimentation. La plupart des habitants de la ville sont approvisionnés par leur famille (parents, grands parents) dans les villages. On estime, par exemple, que la part du circuit privé dans la consommation de viande est de 20 à $30 \%$ environ. Dans la plupart des villages, l'approvisionnement en lait s'effectue à travers le circuit privé. Les spécialistes biélorusses estiment que $80 \%$ de la dose interne provient de la consommation de produits de base comme le lait et les pommes de terre, le reste provenant de la consommation de produits de la forêt qui sont généralement contaminés et officiellement interdits à la consommation.

Cette situation est préoccupante pour les enfants en bas âge qui, faute d'autres produits de remplacement (par exemple les petits pots pour bébé qui ont disparu du marché), sont nourris essentiellement avec du lait. La plupart des villages sont aujourd'hui à nouveau approvisionnés en lait par la production privée. Il faut noter que le circuit privé échappe presque totalement au contrôle public. Les contrôles, d'ailleurs facultatifs, dans le circuit privé ne sont plus pratiqués par la population.

\subsection{Le programme de contre-mesures}

La première ligne de contre-mesures engagées par les autorités a concerné la décontamination des lieux et objets d'usage courant. Il s'agit ici des actions de contrôle et de décontamination des infrastructures publiques (décontamination des hôpitaux, écoles, magasins, goudronnage de routes, etc.) et privées (décontamination des maisons, bâtiments annexes, potagers, etc.). Cette action s'effectue très progressivement en fonction des budgets disponibles, avec une priorité, semble-t-il, pour la ville de Tchetchersk. Nous ne disposons pas d'éléments chiffrés permettant d'évaluer précisément l'état d'avancement de cette action. D'une façon générale, les autorités locales semblent avoir privilégié les interventions sur les infrastructures collectives dans les sites à forte densité démographique. La faible densité des zones rurales du district et particulièrement des zones les plus contaminées (49 habitants en moyenne par localité 
rurale dans la zone 3 dont la contamination se situe entre 15 et $40 \mathrm{Ci} / \mathrm{km}^{2}$ ) contribue fortement à diluer et donc à limiter l'efficacité des mesures.

La seconde ligne d'action est la réduction de la contamination alimentaire lors de la production. Celle-ci passe d'abord par le gel des terrains agricoles les plus contaminés, par l'interdiction de la consommation des produits forestiers, par la sélection de pâturages peu contaminés avec une préparation spéciale des sols (ensemencement avec des variétés sélectionnées, apport d'engrais), par l'arrêt des productions les plus sensibles à la contamination (abandon de la culture du lin et du sarrasin, restriction de l'élevage à l'air libre), par la reconversion vers des cultures adaptées à cet environnement (produits peu contaminés avant ou après transformation agroalimentaire). La réduction de la contamination passe également par des mesures agrochimiques : apport de substances décontaminantes dans l'alimentation des vaches (" cesium binder »), ainsi que par différentes pratiques pour réduire le niveau de contamination des produits avant leur consommation (approvisionnement des bêtes en fourrage non contaminé 3 mois avant l'abattage, sélection des sous-produits du lait les moins contaminés, etc.).

La troisième ligne d'action concerne l'allocation de subventions pour permettre aux enfants résidant sur les territoires contaminés de faire des séjours de vacances hors des territoires contaminés, surtout à l'étranger. De fait, de nombreux enfants rencontrés sur le territoire du district, dans le cadre des entretiens, ont fait un ou plusieurs séjours à l'étranger (Allemagne et Italie principalement). Cette mesure est très populaire.

La quatrième ligne d'action concerne le renforcement des programmes médicaux de contrôle et de soin : visites médicales, acquisition de matériel pour mesurer la contamination interne, acquisition de matériel médical ne faisant pas l'usage de rayons $X$ (pour ne pas augmenter l'exposition), etc. Les entretiens réalisés dans le district montrent que le suivi médical de la population semble assuré, mais surtout en ville. Les personnes interrogées font généralement référence à une ou plusieurs visites médicales annuelles, surtout pour les enfants et les adolescents et plus fréquemment dans la ville de Tchetchersk. En revanche, les infrastructures hospitalières sont fortement délabrées.

D’une façon générale, les habitants de la ville sont très sensibles à la propreté de leur environnement qu'ils associent à la propreté radiologique. Les inquiétudes sur la contamination sont souvent associées à l'idée de « saleté de l'environnement ». On ne s'étonnera donc pas que la municipalité mette particulièrement l'accent sur le lavage, la propreté et l'embellissement de la ville(1).

(1) La municipalité de la ville de Tchetchersk projette la création d'une laverie municipale. Cette mesure, qui peut surprendre a priori, rencontre un écho très favorable au sein de la population. De même, la municipalité de Tchetchersk apporte un soin particulier à l'entretien des abords de la ville et des rues, à la propreté et à l'embellissement de la ville, qui est particulièrement atypique dans le contexte actuel de la Biélorussie. 
Ces mesures n'ont pas d'impact sur la situation radiologique. Elles sont perçues favorablement par les habitants et contribuent à développer chez eux une perception positive de leur environnement et de l'avenir de leur ville.

\subsection{Le contrôle radiologique}

Le contrôle radiologique dans le district de Tchetchersk est assuré par deux organismes : le Centre vétérinaire qui dépend du Ministère biélorusse de l'agriculture d'une part et le Laboratoire sanitaire et épidémiologique qui dépend du Ministère biélorusse de la santé, d'autre part. Ces unités dépendent de l'administration nationale mais le recrutement des cadres est assuré par les autorités locales, ce qui revient à dire qu'elles ne sont pas indépendantes des autorités locales.

Le Centre vétérinaire assure le contrôle de la production, dans les sovkhozes et les kolkhozes. Ces contrôles ne sont régulièrement réalisés (chaque mois) que dans certains centres selon la disponibilité des équipes. Les centres de production ont, en principe, un responsable disposant de compétences en radiologie mais ne sont pas équipés avec le matériel nécessaire pour réaliser sur place les contrôles des produits. Lors de leur passage, les équipes vétérinaires font, s'il y a lieu, des évaluations et des propositions pour identifier l'origine de la contamination et réduire celle-ci. Les mesures proposées n'ont cependant que valeur de recommandation. Les centres de production ne sont pas tenus de les mettre en œuvre, car le centre vétérinaire, tout comme les autorités vétérinaires régionales (Gomel) ne disposent pas du pouvoir nécessaire pour imposer une modification des processus de production.

Sur un plan administratif, les centres de production dépendent du centre administratif du district qui lui-même dépend du centre administratif de la région qui n'ont aucune obligation d'information vis-à-vis de l'autorité vétérinaire. Les autorités vétérinaires indiquent qu'elles ont souvent les plus grandes difficultés à obtenir des centres de production l'application de leurs recommandations.

Le laboratoire d'épidémiologie assure le contrôle du circuit de distribution ainsi que le contrôle des produits de la production privée (à l'initiative de la population qui vient porter ses échantillons de son propre gré). Il y a 6 magasins dans la ville de Tchetchersk et un magasin dans la plupart des villages. Aujourd'hui les magasins de village ne sont plus contrôlés, faute d'essence, mais également faute de produits (crise économique).

Dans les années qui ont suivi l'accident, de nombreux habitants venaient faire contrôler gratuitement leurs produits. Aujourd'hui les demandes d'analyse sont plus rares. Les produits forestiers sont généralement les plus contaminés ainsi que le lait, le gibier ou le poisson. Le laboratoire réalise parfois des contrôles à domicile à la demande de l'hôpital lorsque celui-ci identifie un niveau de contamination particulièrement important chez un individu. 


\subsection{Une nécessaire évolution du cadre législatif}

Le dispositif législatif de 1991 introduit différentes contraintes lourdes concernant la gestion des territoires contaminés qui garantissent un niveau de protection radiologique de la population mais entravent le redéploiement économique des territoires contaminés. Les revendications des autorités locales et régionales concernent la levée de ces contraintes qu'elles considèrent comme rigides et peu adaptées à une gestion fine de la situation sur le terrain : remplacement des critères de contamination du sol par des critères de dose, révision des cartes de contamination pour tenir compte des évolutions de la situation radiologique depuis 86 , autorisation de certaines catégories de production agricole sur les territoires contaminés, révision à la hausse des normes de protection radiologique.

Les autorités nationales sont tenues de mener la gestion post-accidentelle dans le cadre législatif biélorusse. Les contraintes introduites par la loi sont parfois incompatibles avec les objectifs affichés par les autorités régionales et locales. On décèle des tensions entre les différents niveaux de responsabilité administrative chargés de la mise en œuvre des contre-mesures. Il faut rappeler que ces contraintes législatives ont été introduites en phase post-accidentelle dans un contexte de crise sociale et politique et dans un climat de forte suspicion(1) vis-à-vis des experts et des autorités. Ainsi, la référence à la contamination des sols présente un caractère d'objectivité pour la population que ne revêt pas un calcul de dose assis sur un modèle mathématique nécessairement complexe donc opaque pour la population. De même, la référence aux normes internationales apporte à la population des éléments de garantie sur son niveau de protection.

Le contexte économique et politique est aujourd'hui très différent de ce qu'il était en 1990. L'importance de l'inflation a souvent rendu dérisoires les primes perçues par les habitants pour s'approvisionner en produits non contaminés. Cependant, dans un contexte de pénurie, le travail sous contrat qui permet le doublement du salaire apparaît comme un attrait important. Notons encore le caractère improductif, voire contre-productif, de certaines dispositions légales vis-à-vis de la protection radiologique de la population (par exemple le programme de pseudo-relogement dans la ville de Tchetchersk).

Pour différentes raisons donc, le cadre législatif post-accidentel biélorusse apparaît largement inadapté aux enjeux de la réhabilition des territoires contaminés. Il apporte néanmoins des garanties fortes sur un certain niveau de protection. Supprimer certaines de ces garanties implique la création de nouvelles

(1) Cette crise de confiance résultait elle-même de la façon dont la gestion accidentelle avait été conduite (non protection de la population en phase $\mathrm{A}$, censure et secret sur les conséquences de l'accident). 
garanties d'une autre nature. Ceci d'autant plus que les autorités et les experts locaux qui dépendent de ceux-ci sont tentés de privilégier les impératifs économiques, parfois au détriment de la logique de radioprotection.

\section{La situation financière et économique du district de Tchetcherk}

\subsection{Le budget du district}

En 1994, le budget de fonctionnement du district de Tchetchersk est évalué à 7137 millions de roubles. Il est financé à $56 \%$ par des ressources locales (impôt sur les salaires, TVA, revenus forestiers, etc) et à $44 \%$ par des subventions provenant du budget régional. Les principaux postes de dépense de ce budget sont l'éducation ( $28 \%)$, la santé $(25 \%)$, les subventions économiques $(28 \%)$ et la voirie $(17 \%)$.

En plus de ce budget, le district de Tchetchersk dispose d'une subvention nationale allouée dans le cadre du budget Tchernobyl (par le Comité d'État Tchernobyl). Pour le premier semestre de 1994, cette subvention s'est élevée à 4085 millions de roubles. Cette subvention annuelle est donc supérieure au budget annuel de fonctionnement du district.

De 1991 à 1993, la priorité a été donnée au relogement. Ainsi, dans le passé, les sommes consacrées depuis l'accident au relogement ont permis à près de la moitié des familles de Tchetchersk d'acquérir un logement supplémentaire (pour eux-mêmes ou pour leurs enfants) dans un autre district, sans pour autant abandonner leur habitation de Tchetchersk.

Le relogement ne semble plus aujourd'hui être considéré par les autorités du district comme une priorité. Les interviews réalisées sur le terrain en juillet 94 montrent que, pour différentes raisons, la population ne semble plus considérer l'objectif d'un départ comme prioritaire(1).

Les autorités locales ont donc établi, en accord avec les autorités régionales et nationales, un certain nombre d'actions pour tenter d'améliorer les conditions de vie des habitants du district. D'après les autorités locales, près de $35 \%$ du budget Tchernobyl du district est aujourd'hui consacré au paiement de doubles salaires aux populations sous contrat, $38 \%$ à l'alimentation gratuite des écoliers, $9 \%$ aux primes de vacances des personnes résidant sur les zones

(1) Cette attitude contraste fortement avec la situation ukrainienne sur les territoires contaminés (étudiée en 1993), où la population comme les autorités semblent convaincues de la nécessité de reloger tous les habitants des territoires dont le niveau de contamination est supérieur à $15 \mathrm{Ci} / \mathrm{km}^{2}$. 
contaminées. Le reste est consacré aux compensations de biens immobiliers des relogés, au transport de leur mobilier, enfin, pour une très faible part, aux compensations pour invalidité. Seule l'alimentation gratuite des enfants à l'école apporte une contribution passagère à la protection radiologique (apport de nourriture saine et non consommation de production domestique plus contaminée).

\subsection{Une économie agricole fortement perturbée}

La mise en application des mesures de relogement d'une part et des mesures de gel des terres contaminées, d'autre part, a profondément bouleversé l'économie du district, essentiellement agricole. Entre 1988 et 1992, la production du district a chuté de $40 \%$. Depuis 1986, le cheptel a diminué de $40 \%$. Certaines productions ont été abandonnées du fait de la contamination (productions qui concentrent la contamination : lin, sarrasin). Le nombre de vaches laitières dans le district a été divisé par deux, celui des porcs a été réduit de $75 \%$. La main-d'ceuvre agricole du district a diminué de près de $50 \%$ entre 1986 et 1992, en passant de 7400 personnes à 3800 personnes.

Aujourd'hui, le district de Tchetchersk produit essentiellement des pommes de terre, du seigle, du blé, ainsi que du lait et de la viande. On trouve également dans le district quelques petites unités de conserverie, mais très vétustes. Le district compte aujourd'hui environ 37000 hectares de terres cultivées dont les deux/tiers sont situés dans des zones dont la contamination se situe entre $5 \mathrm{Ci} / \mathrm{km}^{2}$ et $40 \mathrm{Ci} / \mathrm{km}^{2}$ (plus de 10000 hectares de terres sont « gelés », car contaminées à plus de $40 \mathrm{Ci} / \mathrm{km}^{2}$ ).

Une partie de la production du district, dont le niveau de contamination n'est pas conforme aux normes alimentaires biélorusses, est exportée vers la Russie où les normes sont moins rigoureuses. La norme pour le lait est par exemple de $111 \mathrm{~Bq} / 1$ (césium) et $3,7 \mathrm{~Bq} / 1$ (strontium) en Biélorussie, alors qu'elle est de $370 \mathrm{~Bq} / \mathrm{l}$ (césium) et $37 \mathrm{~Bq} / \mathrm{l}$ (strontium) en Russie.

Il y a 13 centres de production ( 8 kolkhozes, 4 sovkhozes et un centre de production expérimental) dans le district. La réduction de la main-d'œuvre de ces centres ne s'est pas opérée de façon uniforme sur le territoire. Certains centres de production ont été fortement touchés par cette émigration en perdant jusqu'à $75 \%$ de leur main-d'œuvre (kolkhoze Kirov). D'autres centres ont pratiquement conservé une main-d'œuvre identique à celle de 1986 (sovkhozes Botvinovo et Merkoulovitchski). La figure 7 présente le pourcentage des départs de population des territoires affectés pour les différents centres de production du district. Les différences constatées sont liées aux caractéristiques de la contamination (surface, niveau) qui sont très inégales dans les différents centres de production. On peut également penser que les mouvements de population ont pu être variables selon les villages considérés. 


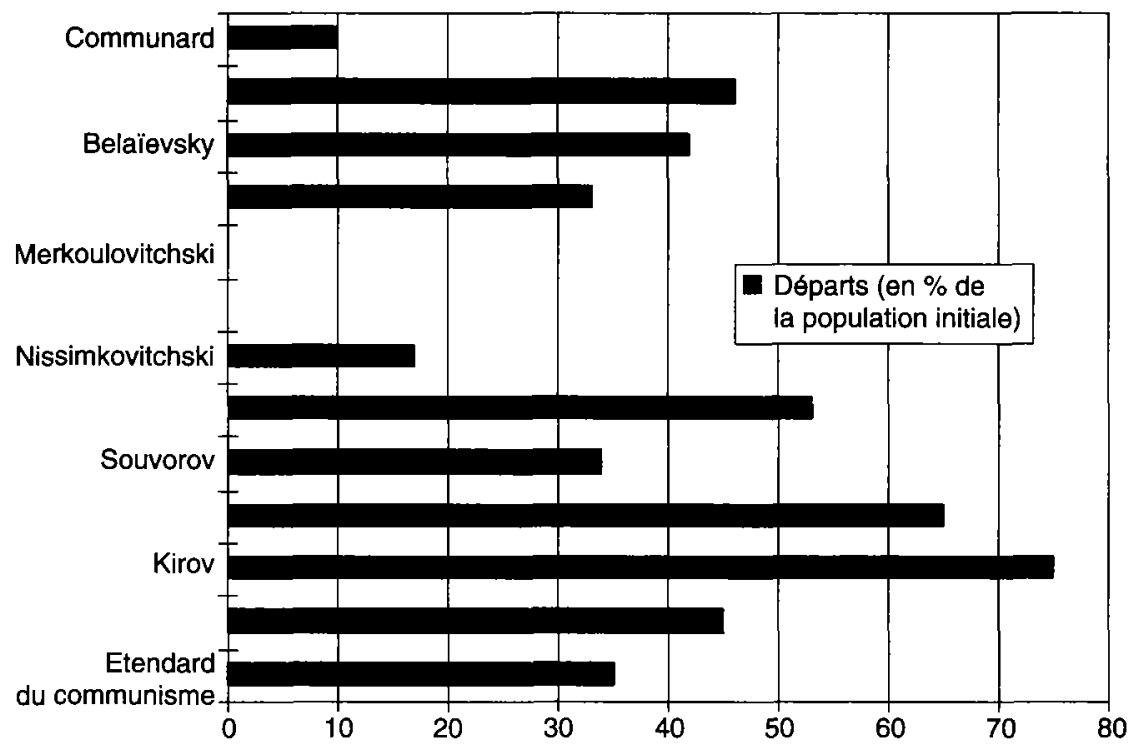

Fig. 7. - Départ de population dans les centres de production agricole du district de Tchetchersk (en pourcentage de la population initiale).

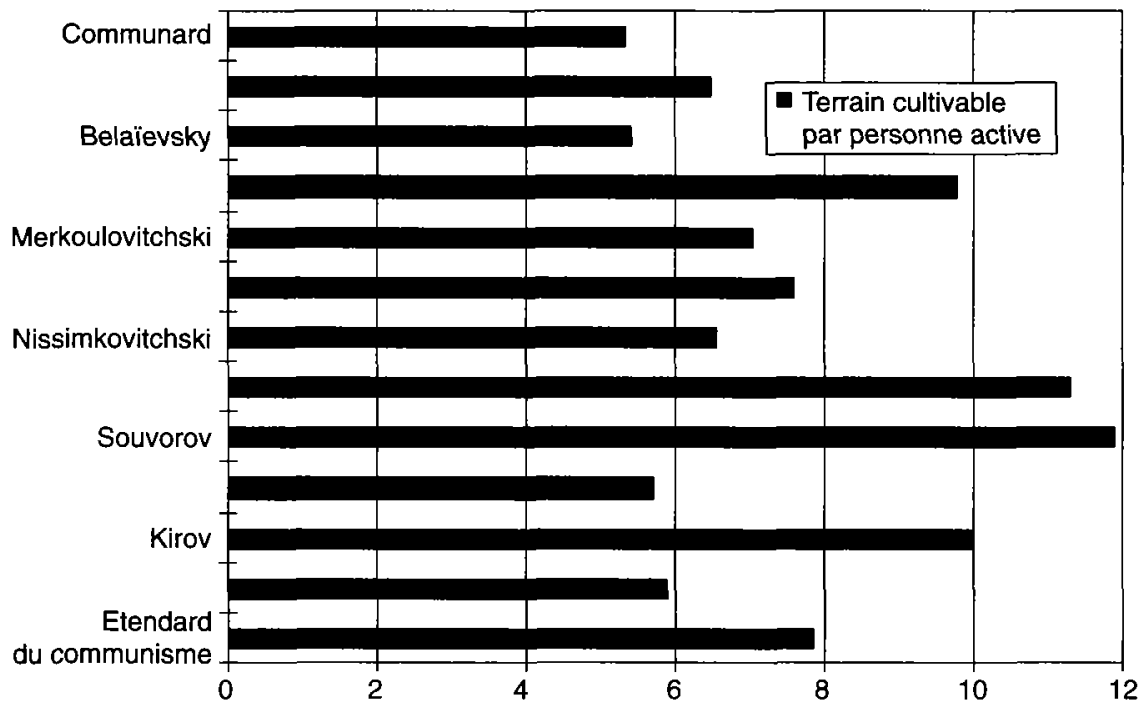

Fig. 8. - Comparaison des surfaces cultivables (en hectares) par personne active dans les centres de production du district de Tchetchersk. 
Parallèlement, la mise en application de la loi sur le statut des territoires contaminés a entraîné le gel de $24 \%$ des terres agricoles du district. Cette mise en jachère a été réalisée en fonction du niveau de contamination et donc de façon inégale selon les centres de production. Dans certains cas, la diminution du nombre d'hectares cultivés par un centre de production s'est accompagnée d'une réduction de la main-d'œuvre habitant sur le territoire du centre. Ainsi, le kolkhoze Lenine a perdu environ $35 \%$ de sa surface cultivable (2 500 ha « gelés » sur un total de 7000 ha) tandis que sa population a diminué de $53 \%$ en passant de 1922 personnes en 1986 à 901 en 1993.

Dans bien des cas, un profond déséquilibre est apparu entre la quantité de main-d'œuvre et la capacité de production (surface cultivable), comme le montre le schéma ci-dessous. Ainsi, par exemple, dans le kolkhoze Lenine, la surface à cultiver par personne active est de 11,3 ha, tandis qu'elle est de 6,5 ha par personne active dans le sovkhoze Nissimkovitchski (Fig. 8).

\section{Conclusion}

La mise en œuvre du programme initial de gestion post-accidentelle se heurte à plusieurs types de difficultés, au plan social, économique et radiologique. Ce programme était fortement axé sur le déplacement des populations des territoires les plus contaminés. Une partie importante des ressources disponibles a donc été affectée au relogement de la population des territoires contaminés. Aujourd'hui dans certains territoires initialement destinés à une évacuation complète, la population s'est en majorité stabilisée et ne semble plus disposée à partir. Des familles initialement relogées sont revenues dans leur lieu de résidence initial, notamment du fait des contraintes économiques (chômage, inflation, pénurie alimentaire).

La présence désormais définitive de populations dans des environnements durablement contaminés pose une nouvelle catégorie de problèmes qui échappaient à la logique initiale de gestion post-accidentelle. Les infrastructures et services publics de ces territoires, fortement délabrés, car promis à l'abandon, doivent aujourd'hui être remis dans un état de fonctionnement satisfaisant. De même un redéploiement économique est aujourd'hui nécessaire dans des régions dont l'activité, principalement agricole, est fortement perturbée par les contraintes radiologiques. Sur ce plan, la situation reste préoccupante. On observe dans ces territoires une régression de la protection radiologique, notamment du fait du redémarrage de la production alimentaire privée qui avait été interrompue après l'accident. D'une façon générale, on observe que les moyens affectés à la gestion post-accidentelle ne contribuent que faiblement au développement de la sécurité radiologique des habitants des territoires les plus contaminés (qu'ils étaient initialement censés quitter).

Ce contexte introduit une nouvelle étape de la gestion post-accidentelle en Biélorussie, dans laquelle les objectifs de radioprotection doivent être resitués 
dans un ensemble de contraintes de différentes natures, notamment sociales et économiques. Le développement de la sécurité radiologique est indissociable du retour d'une qualité de vie dans ces territoires, d'une part. Il passe nécessairement par l'instauration d'un climat de confiance sociale qui fait défaut aujourd'hui (Girard et Hériard Dubreuil, 1995), d'autre part. Mis en place dans le contexte fortement émotionnel de la crise post-accidentelle, le dispositif législatif de 1991 apportait des garanties fortes dans un cadre institutionnel rigide et centralisé. Ce cadre apparaît devoir être adapté pour donner plus d'autonomie aux acteurs locaux dans la reconstruction de conditions de vie satisfaisantes dans ces territoires. Cette autonomie suppose l'instauration de nouvelles formes de régulation qui viendraient garantir que le redéploiement économique et social ne s'effectue pas au détriment de la sécurité radiologique.

\footnotetext{
Remerciements : Les auteurs remercient le $D^{r}$ Igor Rolevitch, ministre adjoint (Ministère biélorusse pour la protection de la population contre les conséquences de l'accident de Tchernobyl). Ils remercient Jacques Lochard du CEPN pour sa généreuse contribution ainsi que Thierry Schneider du CEPN. Ils remercient Jacques Véron, de l'Institut national des études démographiques (INED), d'avoir accepté de faire une lecture critique de cet article avec de nombreuses observations et suggestions qui ont beaucoup contribué à la qualité de ce travail. Ils remercient enfin Neale Kelly et Jaak Sinnaeve de la Commission européenne (DG. XII) pour leur soutien constant au cours de cette recherche.
}

\section{RÉFÉRENCES}

Girard P. et Hériard Dubreuil G. (1994) Conséquences sociales et psychiques de l'accident de Tchernobyl. La situation en Ukraine, 7 ans après l'accident, Juillet 1994 (MUTADIS Consultants, Paris, REF MUT 93/JSP2 $\left.{ }^{\circ} \mathrm{PG} / \mathrm{GHD} / 003\right)$.

Girard P., Hériard Dubreuil G. (1995) Conditions de vie dans les territoires contaminés 8 ans après l'accident de Tchernobyl, Gestion du risque radiologique en Biélorussie : évaluation de la situation dans le district de Tcherchersk, Juin 1995, (MUTADIS Consultants Paris, référence : MUT 95/JSP2/PG/GHD/003).

Hériard Dubreuil G. (1994) Un premier bilan des effets psychiques et sociaux de l'accident de Tchernobyl. Radioprotection, 29, 363. 